\title{
MODELO COGNITIVO METODOLÓGICO PARA LA GENERACIÓN Y APORTES DE RESULTADOS CIENTÍFICOS
}

\author{
George ARGOTA PÉREZ \\ Centro de Investigaciones Avanzadas y Formación Superior en Educación, \\ Salud y Medio Ambiente "AMTAWI". Puno, Perú. \\ george.argota@gmail.com \\ Rosalvina CAMPOS PÉREZ \\ Facultad de Humanidades. Universidad Nacional Federico Villarreal. Lima-Perú \\ rosalvina17@gmail.com \\ José IANNACONE \\ Laboratorio de Parasitología. Facultad de Ciencias Biológicas. \\ Universidad Ricardo Palma. Lima-Perú. \\ jose.iannacone@urp.edu.pe \\ Javier CHÁVEZ ESPINOZA \\ Facultad de Farmacia y Bioquímica. Universidad Nacional San Luis Gonzaga. Ica-Perú \\ qfjche@gmail.com

\section{América SOTO CÁRDENAS} \\ Facultad de Farmacia y Bioquímica. Universidad Nacional San Luis Gonzaga. Ica-Perú \\ americascg@hotmail.com \\ Patricia CASTILLO ROMERO \\ Facultad de Farmacia y Bioquímica. Universidad Nacional San Luis Gonzaga. Ica-Perú \\ pccrfar@hotmail.com
}

\section{RESUMEN}

La tenencia de modelos integrales que permitan dimensionar nuevos conocimientos continúa siendo un reto a demostrar. El propósito del estudio fue describir un modelo cognitivo metodológico para la generación y aportes de resultados científicos. Mediante la técnica cuantitativa por análisis de contenido se construyó el modelo propuesto el cual consistió en tres procesos: $1^{\text {ro }}$ ) gestión de la información científica, $2^{\mathrm{do}}$ ) interpretación cognoscitiva; y $3^{\mathrm{ro}}$ ) redacción científica. Cada proceso deriva a estructuras de reconocimientos de forma transicional lo que posibilita, la construcción hacia una información validada. Se concluyó que, en cualquier campo de la investigación se requiere desde la captura de la información primaria hasta la nueva comunicación científica lograda por cuanto, así lo permitió el modelo propuesto.

\section{PALABRAS CLAVE}

Ciclo de la investigación, cognición metodológica, diseño metodológico, investigación científica 


\title{
METHODOLOGICAL COGNITIVE MODEL FOR THE GENERATION AND CONTRIBUTIONS OF SCIENTIFIC RESULTS
}

\begin{abstract}
The possession of integral models that allow to dimension new knowledge continues being a challenge to demonstrate. The purpose of study was to describe a methodological cognitive model for the generation and contribution of scientific results. Through the quantitative technique by content analysis, the proposed model was constructed, which consisted of three processes: $1^{\text {st }}$ ) management of scientific information, $2^{\text {nd }}$ ) cognitive interpretation; and $3^{\text {rd }}$ ) scientific writing. Each process derives recognition structures in a transitional manner, which makes it possible to, the construction towards a validated information. It was concluded that, in any field of research, it is required from the capture of primary information until the new scientific communication achieved as far as the proposed model allowed.
\end{abstract}

\section{KEY WORDS}

Research cycle, methodological cognition, methodological design, scientific research

Recibido: 08/04/2019

Aprobado: 03/06/2019

\section{INTRODUCCIÓN}

L

a aceptación de los resultados científicos constituye en sí misma, un grave problema, pues no basta, valorar el cumplimiento de estructuras metodológicas sino, el reconocer la validación sobre cada información declarada. Steel y Whyte (2012) y Elliott (2016) señalan que, comprender la simplicidad, capacidad de explicación, generalización de fenómenos, selección de pruebas, novedad y el rigor científico entre otras razones sin duda alguna, determinan lo pretendido para aportar nuevos juicios de valor en cualquier campo de investigación. La esencia común sobre lo deseado radica en la integridad de la investigación científica (De Winter y Kosolosky, 2013), además, del esfuerzo democrático sobre lo que se requiere informar (Schroeder, 2017).

Sin embargo, entender la integridad de la investigación científica se requiere reconocer que, la misma funciona como una red compleja donde los objetos están conectados multi-relacional de forma directa e indirecta (Grabowicz et al., 2012; Ströele, Zimbrão y Souza, 2013) y las agrupaciones que se generan, obedecen a una razón no trivial. En tal sentido, el modelo teórico de la gestión del ciclo de vida de la información de las investigaciones pedagógicas propuesto por Gorina y Alonso (2017), evidencia que existe un flujo informacional para el análisis sistémico de las investigaciones (Fig. 1). A pesar de lo señalado, pudiera considerarse con cierta dificultad que, generar información novedosa comienza con plantear el problema científico (PC) pero quizás no sea así, pues previamente debe dotarse de herramientas científicas para entender que el PC principal, es el anunciado y no otro. 
El propósito del estudio fue describir un modelo cognitivo metodológico para la generación y aportes de resultados científicos.

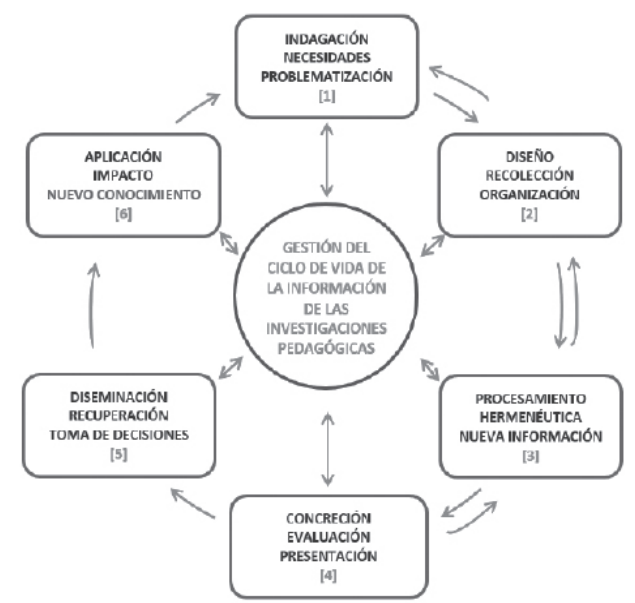

Figura 1. Modelo teórico de la gestión del ciclo de vida de la información de las investigaciones pedagógicas (Gorina y Alonso, 2017).

\section{MATERIALES Y MÉTODOS}

La descripción del modelo cognitivo metodológico para la generación y aportes de resultados científicos se basó en la técnica cuantitativa por análisis de contenido. Algunas referencias consultadas sirvieron de base para la construcción de los tres procesos: $1^{\text {ro }}$ ) gestión de la información científica, $2^{\mathrm{do}}$ ) interpretación cognoscitiva; y $3^{\text {ro }}$ redacción científica (tabla 1).

Tabla 1. Fuentes teóricas de relaciones semánticas / investigación científica.

\begin{tabular}{lr}
\hline \multicolumn{1}{c}{ Contenido } & Fuente \\
\hline $\begin{array}{l}\text { Analyzing scientific context of researchers and } \\
\text { communities by using complex network and semantic } \\
\text { technologies }\end{array}$ & $\begin{array}{r}\text { Horta, Ströele, Braga, } \\
\text { David y Campos (2018) }\end{array}$ \\
$\begin{array}{l}\text { Qualitative research is a fundamental scientific process } \\
\text { Predicting rank for scientific research papers using }\end{array}$ & $\begin{array}{r}\text { Sale y Thielke (2018) } \\
\text { supervised Learning }\end{array}$ \\
$\begin{array}{l}\text { How scientific researchers form green innovation } \\
\text { behavior: An empirical analysis of China's enterprises }\end{array}$ & $\begin{array}{r}\text { y Safi (2018) } \\
\text { Li, Wuan y Wu (2018) }\end{array}$ \\
\hline
\end{tabular}

Aspectos éticos: los autores declaran que se cumplieron todos los aspectos éticos nacionales e internacionales. 


\section{RESULTADOS}

El modelo cognitivo metodológico para la generación y aportes de resultados científicos consistió en los 3 procesos previamente mencionados y donde se reconoció, otras estructuras de reconocimiento (Fig. 2).

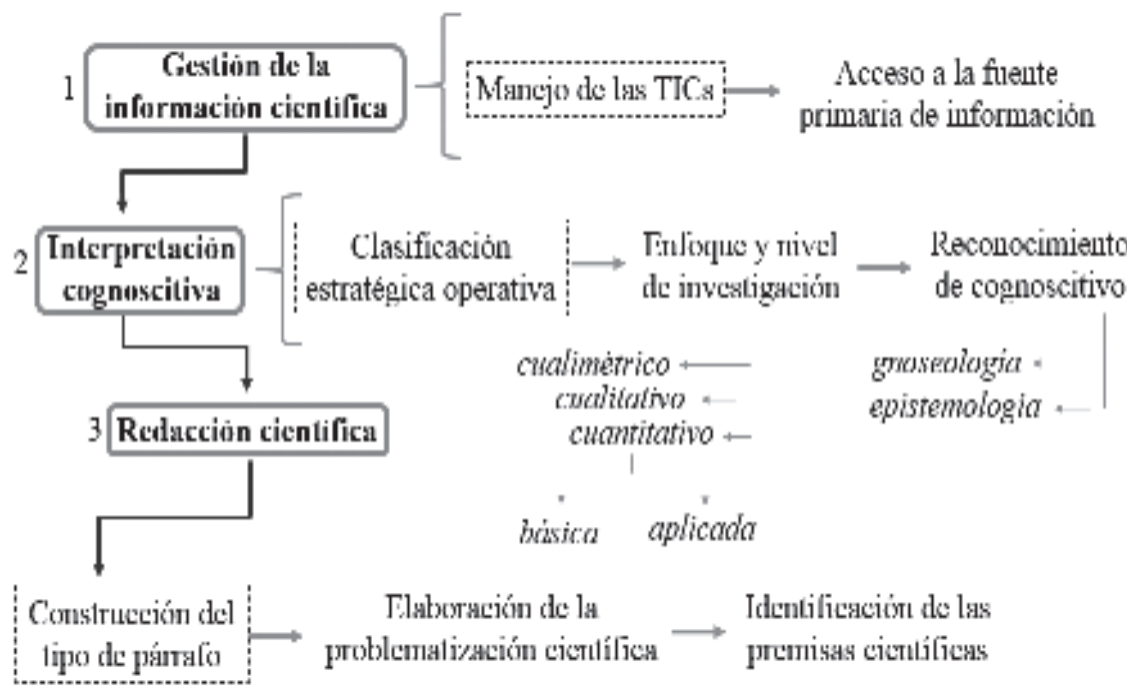

Figura 2. Modelo cognitivo metodológico para la generación y aportes de resultados científicos.

\section{DISCUSIÓN}

Existe una variedad de enfoques que caracterizan los fenómenos a investigar y donde pretenden el análisis desde una perspectiva u orientación multinivel (Geels, 2010; Smith et al., 2010; de Haan y Rotmans, 2011) siendo similar, al modelo cognitivo metodológico para la generación y aportes de resultados científicos que se elaboró. La misma dinámica en sus tres procesos identificados coincidió con lo señalado por Smith et al. (2005) cuando exhortaron que, debe existir transición en el análisis de contexto. Cada uno de los procesos cumple y caracteriza el nivel de reconocimiento y con ello, el aporte aditivo según el criterio potencial de la investigación que se muestre como interés. Asimismo, el modelo propuesto indicó en el segundo proceso su comprobación como sistema de innovación tecnológica de acuerdo en determinado momento, al nivel de investigación (con enfoque cuantitativo) lo que coincidió con lo planteado por Markard y Truffer (2008) y Jacobsson y Bergek (2011) donde señalaron que, la evolución sobre algún resultado pretendido debe gestionarse desde su acceso informacional, reconocimiento cognoscitivo hasta la comunicación científica para cualquier escrutinio. 
Autores como van den Bergh et al. (2006) y Safarzynska y van den Bergh (2012) indican que, el planteamiento de teorías evolucionadas es lo que demuestra, cuánto se progresa o aporta en el proceso de investigación científica de manera que, la elaboración sobre la problematización y construcción de premisas científicas señaladas en el modelo obtenido, posibilita reconocer cualquier regla, principio, ideas que expliquen un fenómeno, deducción producto de la observación siguiéndose un razonamiento lógico.

Se concluyó que, la descripción del modelo permitió la cognición metodológica para la generación y aportes de resultados científicos en cualquier campo básico y de aplicación tecnológica debido a, su concepción desde la captura de la información primaria hasta la obtención de la nueva comunicación científica.

\section{REFERENCIAS BIBLIOGRÁFICAS}

De Haan, J. y Rotmans, J. (2011). Patterns in transitions: understanding complex chains of change. Technological Forecasting and Social Change, 78, 90-102.

De Winter, J. y Kosolosky, L. (2013). The epistemic integrity of scientific research. Science and Engineering Ethics, 19, 757-774.

El Mohadab, M., Bouikhalene, B. y Safi, S. (2018). Predicting rank for scientific research papers using supervised learning. Applied Computing and Informatics, 15, 182-190.

Elliott, K. (2016). Standardized study designs, value judgments, and financial conflicts of interest in research. Perspectives on Science, 24, 529-551.

Geels, F. (2010). Ontologies, socio-technical transitions (to sustainability), and the multi-level perspective. Research Policy, 39, 495-510.

Gorina, S.A. \& Alonso, B.I. (2017). Gestión del ciclo de vida de la información de las investigaciones pedagógicas. Revista Varela, 17, 279-296.

Grabowicz, P.A., Ramasco, J.J., Moro, E., Pujol, J.M. y Eguiluz, V.M. (2012). Social features of online networks: The strength of intermediary ties in online social media. PLOS ONE, 7: 1-9.

Horta, V., Ströele, V., Braga, R., David, J.Ma.N. y Campos, F. (2018). Analyzing scientific context of researchers and communities by using complex network and semantic technologies. Future Generation Computer Systems, 89, 584-605.

Jacobsson, S. y Bergek, A. (2011). Innovation system analyses and sustainability transitions: contributions and suggestions forresearch. Environmental Innovation and Societal Transitions, 1:41-57.

Li, G., Wang, X. Wu. J. (2018). How scientific researchers form green innovation behavior: An empirical analysis of China's enterprises. Technology in Society, $56,134-146$. 
Markard, J. \& Truffer, B. (2008). Technological innovation systems and the multi-level perspective: towards an integrated frame-work. Research Policy, 37, 596-615.

Safarzynska, K. \& van den Bergh, J. (2010). Evolutionary modelling in economics: a survey of methods and building blocks. Journal of Evolutionary Economics, 20, 329-373.

Sale, J.E.M. \& Thielke, S. (2018). Qualitative research is a fundamental scientific process. Journal of Clinical Epidemiology, 102, 129-133.

Schroeder, A. (2017). Using democratic values in science: An objection and partial response. Philosophy of Science, 84, 1044-1054.

Smith, A., Stirling, A. \& Berkhout, F. (2005). The governance of sustainable sociotechnical transitions. Research Policy, 34, 1491-1510.

Smith, A., Voß, J.P. \& Grin, J. (2010). Innovation studies and sustainability transitions: the allure of the multi-level perspective and its challenges. Research Policy, $39,435-448$.

Steel, D. \& K. Whyte. (2012). Environmental justice, values, and scientific expertise. Kennedy Institute of Ethics Journal, 22, 163-182.

Ströele, V., Zimbrão, G. y Souza, J.M. (2013). Group and link analysis of multi-relational scientific social networks. Journal of Systems and Software, 86, 1819-1830.

Van den Bergh, J., Faber, A., Idenburg, A. y Oosterhuis, F. (2006). Survival of the greenest: evolutionary economics and policies for energy innovation. Environmental Science \& Technology, 3, 57-71. 